\title{
Perfluoro-n-octane as a temporary intraocular tamponade in a staged approach to manage complex retinal detachments
}

This article was published in the following Dove Press journal:

Clinical Ophthalmology

27 February 2015

Number of times this article has been viewed

\section{Daniel Barthelmes Jay Chandra}

Department of Ophthalmology, Westmead Hospital, Westmead, NSW, Australia
Correspondence: Jay Chandra Department of Ophthalmology, Westmead Hospital, Darcy Road, Westmead, NSW 2145, Australia Tel +6I 298455509

Fax +6I 298456668

Email chandraeye@hotmail.com
Purpose: To evaluate outcomes in patients with complex retinal detachments (RD) with proliferative vitreoretinopathy (PVR) requiring retinectomy using a staged approach utilizing perfluoro-n-octane (PFO) as a short-term postoperative intraocular tamponade.

Methods: Retrospective analysis. Patients who underwent 23G pars plana vitrectomy for the management of complicated RD where PFO was used as a primary temporary intraocular tamponade. Only eyes with PVR in rhegmatogenous RD or eyes with penetrating injuries or globe ruptures (ocular trauma) and subsequent RD with PVR were included. Analysis of 17 eyes of 17 consecutive patients with a minimum follow-up of 12 months during a period of 5 years. The primary outcome measure was the reattachment rate after at least 12 months of follow-up after the PFO removal. Secondary outcome measures were changes in visual acuity, complications due to PFO use, and necessity for further surgeries.

Results: Eight eyes with prior penetrating injuries or globe rupture and nine eyes with rhegmatogenous pathology were included. All eyes had PFO as temporary tamponade for 14 days (median), which was replaced by silicon oil. Sixteen eyes (94\%) had complete and one eye partial reattachment. No redetachments occurred. All eyes retained or improved visual acuity. On average, only 2.5 procedures, including silicon oil removal, were performed. Two eyes had long-term intraocular pressure of $5 \mathrm{mmHg}$ and no eye had intraocular pressure of $\geq 21 \mathrm{mmHg}$. No long-term inflammation was observed.

Conclusion: PFO seems to be beneficial in a staged approach to repair complex detachments when used as short-term tamponade. A median period of 14 days allowed for both sufficient retinal support plus a reduction in side effects seen in long-term endotamponades.

Keywords: pars plana vitrectomy, complex retinal detachments, proliferative vitreoretinopathy, trauma

\section{Introduction}

One of the big challenges in vitreoretinal surgery is management of complex retinal detachments $(\mathrm{RD})$ following trauma or RD with extensive proliferative vitreoretinopathy (PVR). PVR has been reported to occur in up to $11 \%$ of patients with rhegmatogenous $\mathrm{RD}^{1}$ or in the course of penetrating eye injuries and globe ruptures at an estimated incidence of $10 \%-45 \% .^{2}$ A recent report on injured soldiers showed even up to $70 \%$ PVR. $^{3}$

The traction caused by retinal shortening occurs preferably in the inferior quadrant near or in the vitreous base..$^{4-7}$ Management of complex RD includes scleral buckling, pars plana vitrectomy (PPV), retinectomy, removal of proliferative membranes, and the use of perfluorocarbon liquids or silicon oil. Since standard silicon oil provides inappropriate support for inferior retinal pathologies, "heavier than water" endotamponades 
were developed. ${ }^{8}$ These substances were reported to be not suitable for long-term tamponades, such being necessary in cases of PVR. ${ }^{9,10}$ Later, perfluorohexyloctanes also showed complications as a long-term vitreous substitute. ${ }^{11-13}$ Main problems were intraocular inflammation and toxicity. ${ }^{11,14}$ The currently available "heavy" silicon oils such as Densiron $68^{\circledR}$ seem to be better tolerated but have also been reported to show side effects such as intraocular inflammation. ${ }^{15,16}$ A number of recent studies reported on the possibility of using perfluoro-n-octane (PFO) as a short-term endotamponade that is replaced by air, $\mathrm{C} 3 \mathrm{~F} 8$, or silicon oil after a period of 1-2 weeks. ${ }^{17,18}$ So far, the results achieved in giant retinal tears seem to be promising: ie, retinal attachment was achieved with minimal side effects of PFO. ${ }^{17,18}$

The current study describes the use of PFO as a short-term tamponade in a staged approach to manage cases of complex PVR-related RD in eyes with rhegmatogenous RD and eyes suffering from ocular trauma.

\section{Methods}

\section{Patients}

Retrospective analysis of all patients who underwent $23 \mathrm{G}$ PPV at the Department of Ophthalmology, Westmead Hospital, Westmead, NSW, Australia, for the management of complicated RD where PFO (Perfluoron ${ }^{\circledR}$, Alcon Laboratories, Inc., Fort Worth, TX, USA) was used as a primary temporary intraocular tamponade. Observation period was from August 1, 2006 to December 31, 2011. Only eyes were included with no prior vitreoretinal surgery showing PVR in rhegmatogenous detachments or RD with PVR in eyes with penetrating injuries or globe ruptures (ocular trauma). Eyes with other reasons for traction membranes were excluded, as well as eyes with redetachments after previous retinal detachment repairs. A detailed description of routine care for patients undergoing $23 \mathrm{G} \mathrm{PPV}$ at the same hospital was published previously. ${ }^{19}$

The decision to use PFO as a primary short-term endotamponade was made in cases where after successful intraoperative flattening of the retina, no stable situation could be achieved when gas or silicon oil was used: ie, in cases of a high risk of immediate redetachment. A high risk of immediate redetachment was considered in situations where large retinectomies had to be performed and posterior retinal slippage would occur once PFO was removed, extensive inferior pathology or an impossibility to completely remove all traction membranes where the retinal tissue would remain completely attached only under PFO.

\section{Surgical technique}

All eyes included in this study underwent a standard three-port PPV using the Alcon $23 \mathrm{G}$ system (Accurus ${ }^{\circledR}$ $800 \mathrm{CS}$ ). The surgical technique for $23 \mathrm{G}$ PPV was described previously. ${ }^{19}$ Briefly, a complete vitrectomy was performed with scleral depression to improve visualization of the vitreous base and to assist the meticulous removal of the vitreous. If possible, traction membranes were completely removed. In cases where it was not possible to remove the whole traction membrane, a retinectomy was performed to release traction using the $23 \mathrm{G}$ vitrector after applying full-thickness retinal cautery. All retinal tissue anterior to the retinectomy was removed. If a buckle was in place, all retinal tissue anterior to the retinectomy, up to the apex of the buckle, or up to the edge where the retina was firmly attached to the buckle, was removed. The retina was then flattened using PFO. The posterior edges of the retinectomy were treated using an endolaser probe (Oculas, Alcon, Forth Worth, TX, US), with at least three to four rows of laser spots. After successful reattachment of the retina, the eye was completely filled with PFO, and all sclerotomy ports sutured with 7-0 vicryl sutures.

In phakic patients, the crystalline lens was removed before starting the vitrectomy, and in case of an intact capsule an intraocular lens was implanted (Alcon MA50BM): ie, eyes were either aphakic (eg, trauma cases) or pseudophakic before starting the retinal repair. Chandelier systems were used if deemed necessary to facilitate bimanual dissection of retinal membranes or retinal tissue. In cases of extensive lacerations of the globe or extensive inferior PVR, an encircling buckle was put in place in the same surgical session prior to starting the $23 \mathrm{G} P \mathrm{PV}$.

Approximately 2 weeks after the first vitreoretinal surgery, a second $23 \mathrm{G}$ PPV was performed where PFO was completely evacuated from the vitreous cavity and replaced by silicon oil (Oxane 1300, Bausch and Lomb, Macquarie Park, NSW, Australia). All ports were sutured using 7-0 vicryl sutures.

Depending on the initial pathology, the status of the retina, and the patient's visual potential, a third $23 \mathrm{G} \mathrm{PPV}$ was performed to remove the silicon oil from the vitreous cavity approximately 3-4 months after the silicon oil filling. In these cases, the eye was filled with $25 \%$ SF6 gas to prevent postoperative hypotony. Generally, in cases with low visual potential (eg, macular retinal damage) and an estimated high risk of hypotony, the silicon oil was left as permanent endotamponade. 


\section{Data}

Data were retrieved from the patients' records. The information collected included sex; preoperative best-corrected visual acuity (VA, LogMAR [log of the minimum angle of resolution]) before the initial PPV; age; dates of surgeries performed; types of surgeries performed, including buckle surgery; lens status before vitrectomy; and initial pathology/ reason for vitreoretinal repair. Postoperative data analyzed were best-corrected VA during the follow-up phase; final retinal status; final presence of silicon oil endotamponade; complications such as retinal redetachment, corneal opacification, and endophthalmitis; and significant intraocular pressure (IOP) changes. VA was recorded using a Snellen scale and converted to the LogMAR scale. Institutional Review Board/Ethics Committee approval was obtained from the local Ethics Committee at the Westmead Hospital. The study adheres to the tenets of the Declaration of Helsinki.

\section{Statistical analysis}

Statistical analyses were performed using PASW 19 (SPSS Inc., Chicago, IL, USA). Normally distributed data are presented as mean \pm standard deviation; not normally distributed data are presented as median and range (minimum to maximum). Data distribution was assessed using the ShapiroWilk and Kolmogorov-Smirnov tests. Differences in variances were assessed using Levene's test. Depending on data distribution, tests for not normally or normally distributed data were used taking into account possible pairing of samples and inequality of variances. Differences in proportions were assessed using Fisher's exact test. Results are expressed as odds ratio and $95 \%$ confidence interval. IOP was classified as increased if $\geq 21 \mathrm{mmHg}$ with or without glaucoma medication. Hypotony was classified as IOP $\leq 5 \mathrm{mmHg} \cdot{ }^{20,21}$ Corneal haze was graded as "present" or "not present". Statistical significance was defined as $P<0.05$.

\section{Results}

After applying inclusion and exclusion criteria, 17 eyes from 17 patients were available for final analysis from the observation period August 1, 2006 to December 31, 2011 ( $~ 5.3$ years). In all 17 eyes, complete documentation and follow-up data were available. Follow-up time was $37.1 \pm 20.1$ (range 12-64) months. The exact time at which retinal detachment developed could not be determined in most cases, due to patient logistics: ie, referral patterns and primary care in case of trauma. Eyes with trauma are treated immediately by the surgeon on call, not by the vitreoretinal unit. In most cases, vitreoretinal surgery was performed often $7-10$ days after primary repair. In patients with rhegmatogenous detachments, symptoms have been reported to be present for at least 2-3 months.

The 17 eyes were from 12 (71\%) men and five (29\%) women. Mean age at the time of the first surgical intervention was $44.2 \pm 16.2$ years (men $46.1 \pm 16.4$, women $39.8 \pm 16.4$, $P=0.48$ ). A strong imbalance regarding the side affected was found: two (12\%) right eyes and $15(88 \%)$ left eyes were observed. Patients in the group with rhegmatogenous detachments were older (51.3 \pm 5.1 years) as compared with patients with traumatized eyes ( $36.3 \pm 4.9$ years); the difference was not significant $(P=0.051)$.

Eight eyes (47\%) had previous traumatic damage and subsequent development of a retinal detachment with PVR, while nine eyes (53\%) had rhegmatogenous detachments and severe PVR (grade C or D) development. Seven eyes (41\%) were aphakic at the first PPV; all of them had suffered from ocular trauma (globe rupture, penetrating eye injury) that effectuated loss of intraocular tissue, including the crystalline lens. Only one of the eight eyes with trauma could retain the crystalline lens. This was removed with a standard phacoemulsification procedure before the $23 \mathrm{G}$ PPV. No eye with rhegmatogenous RD was aphakic. The preoperative (ie, before the first 23G PPV) VA in all patients was $3.0(0.8-4.0)$ LogMAR, whereas preoperative VA in traumatized eyes was 4.0 (2.0-4.0) LogMAR and in eyes with rhegmatogenous detachments was $3.0(0.8-4.0) \operatorname{LogMAR}(P=0.34)$. Table 1 gives a detailed overview.

All eyes had anterior retinal defects of at least 2 clock hours either due to traumatic damage or due to necessary retinectomies. An encircling buckle was placed in eleven eyes $(65 \%)$, where four of the eight eyes with traumatic damage and seven of the nine eyes with rhegmatogenous retinal detachment had buckle surgery. The difference in proportions in buckle surgery between both groups was not significant $(P=0.33)$.

The time between the PFO filling (first 23G PPV) and the PFO removal/silicon oil filling (second 23G PPV) was 14 (13-28) days.

Complete reattachment of the retina was achieved in 16 of 17 eyes (94\%). In one eye of the group of eyes having suffered from penetrating injury, new subretinal traction bands developed under the silicon oil tamponade, which led to a small redetached area in the inferior fundus. As the macular region and the remaining retina were stabilized, no further interventions were planned and the situation remained stable: ie, no further deterioration or detachment occurred. 


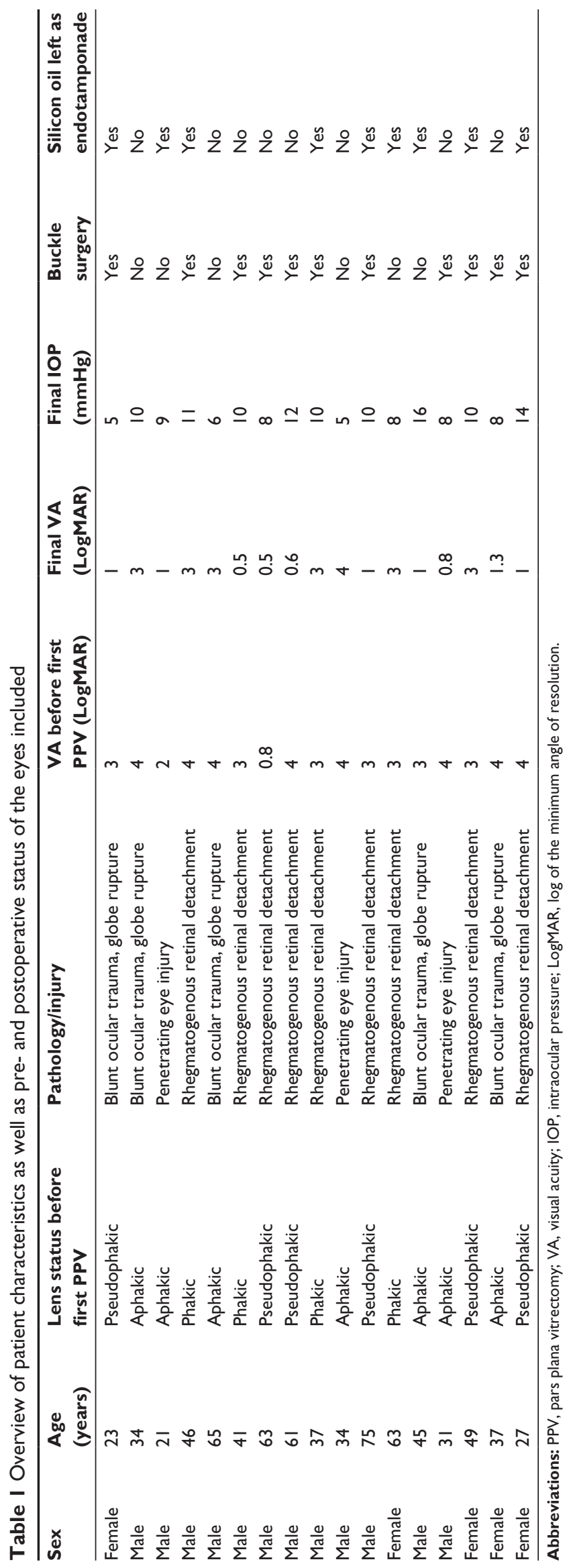

In case the silicon oil was left as a permanent endotamponade, only two 23G PPVs were performed (PFO filling/ PFO removal and silicon oil filling); all other eyes had an additional surgery in which the silicon oil was exchanged for SF6. The median number of $23 \mathrm{G}$ PPVs in all eyes was two. No significant difference was found between traumatized eyes and eyes with rhegmatogenous detachments. In eyes with rhegmatogenous detachments, in only three of nine (33\%) could the silicon oil be removed, while in the eyes with previous trauma, in five of eight (63\%) the silicon oil could be removed $(P=0.35)$. Postoperative VA in all eyes was $1.0(0.5-4.0) \operatorname{LogMAR}$, which is a significant increase compared with preoperative VA $(P=0.0016)$. Postoperative $\mathrm{VA}$ in eyes with previous rhegmatogenous detachments was $1.0(0.5-3.0)$ LogMAR and 1.2 (0.8-4.0) LogMAR in eyes with previous trauma. In both rhegmatogenous detachments ( $P=0.031)$ as well as in eyes with previous trauma $(P=0.021)$, VA significantly increased and no differences in postoperative VA were found between both groups $(P=0.52)$. All eyes either retained or improved VA. There was no difference in the proportion of eyes achieving 1 LogMAR or better between eyes with rhegmatogenous detachments versus those with previous trauma. The best visual recoveries ( 0.6 LogMAR or better) were achieved in eyes with previous rhegmatogenous detachments only.

None of the eyes developed glaucoma. Two eyes were hypotonic (Table 1). All eyes were stable, no redetachments occurred, and in none of the eyes was further surgery or laser photocoagulation required. Three eyes (all three eyes initially had globe ruptures with loss of intraocular tissue and loss of the crystalline lens) developed a hazy cornea during the follow-up period; none of these eyes had a permanent endotamponade with silicon oil. During the PFO endotamponade period, anterior chamber inflammation could be observed in all eyes. This subsided after the PFO removal and, eventually, no eye had persistent intraocular inflammation. As a consequence of the anterior chamber inflammation, five eyes developed posterior synechiae. No endophthalmitis was observed.

\section{Discussion}

To our knowledge, this is the first report on PFO as a primary short-term endotamponade in a staged approach to manage complex retinal detachment in eyes with extensive retinal damage that did not undergo any previous vitreoretinal repair. Complete retinal attachment was achieved in $94 \%$ of eyes, at an overall average of only $2.523 \mathrm{G}$ PPV per eye, including the final silicon oil removal. 
Overall, the results of the current study in respect of reattachment rate (complete reattachment in 94\%) and silicon oil removal rate $(47 \%)$ are comparable with previous results in a similar patient cohort with inferior pathology, PVR, and inferior retinectomy. ${ }^{22}$ The number of additional surgeries after the retinectomy was reported to be, on average, 1.8 including the silicon oil removal, which is slightly higher compared with the currently reported 1.5 surgeries including the silicon oil removal. In contrast to reports analyzing PFO in complex detachments where most of the eyes had already undergone one or more surgeries to reattach the retina, ${ }^{17,22}$ none of the eyes in this study had previous vitreoretinal surgery. Given this, using the described approach, the number of surgeries in complex RD with PVR can be reduced significantly: the primary repair using PFO, the removal of PFO and replacement with silicon oil, and possibly the removal of silicon oil and replacement by gas. Furthermore, using the proposed approach and avoiding extensive retinectomy and aggressive relief of traction membranes - which are factors that may lead to further PVR development and potential final surgical failure - may have contributed to the results reported. Especially in younger patients, such as those with severe injuries and a higher potential for PVR and risk of retinal redetachment under silicone oil, primary use of PFO may be an alternative to achieve stable reattachment.

When using PFO for a short time only (in the current study 14 days [median]), similarly to reports in giant retinal tears, ${ }^{18,23}$ the clear benefits of a sufficient retinal support were combined with a reduction of side effects, as PFO is completely removed after 1-2 weeks. Nevertheless, some of the side effects, such as inflammation or corneal opacification, cannot be completely avoided. Despite corticosteroid medication, some degree of intraocular inflammation could be observed in all eyes during the PFO endotamponade, which may have contributed to the formation of posterior synechiae observed in five eyes. Corneal opacification that occurred in aphakic eyes with previous trauma may be an argument against the usage of PFO as a primary endotamponade. However, as the retina could be reattached in all these eyes and remained stable, it may be possible to perform a keratoplasty later. It may be argued that corneal endothelial damage should be avoided, especially in aphakic or aniridic eyes, since corneal surgery adds additional trauma, and outcomes of corneal transplant procedures are less favorable. However, given the retinal damage, a high risk for final surgical failure and eventual loss of the eye when using gas or silicone oil initially has to be balanced against sequelae of using PFO while still retaining good potential for visual recovery after keratoplasty.

While 14 eyes had a normal IOP, two eyes developed hypotony. These two eyes had penetrating injuries and globe ruptures with loss of the crystalline lens and iris tissue. It is not clear whether the hypotony is the effect of the PFO tamponade ${ }^{24}$ or rather an effect of the damage inflicted to the anterior segment of the eye, including the ciliary body. One could argue that heavy silicon oil may be an alternative to PFO fillings. However, also for Densiron $68^{\circledR}$, for example, side effects such as inflammation and mechanical damage were reported. ${ }^{15,16}$ Another important factor is that the handling of heavy silicon oils is more difficult and takes more time as compared with PFO, especially when using small gauge instruments. While it has been reported that Densiron endotamponades can be managed with $23 \mathrm{G}$ instruments, though using very high suction pressures of $600 \mathrm{mmHg},{ }^{25}$ the ability to inject and remove PFO using a plain syringe with soft-tip cannulas appears more appealing in complex cases such as those described here.

The use of buckling surgery especially in aphakic or pseudophakic eyes may warrant brief discussion. Especially in traumatized eyes, adding an encircling buckle may add to orbital trauma and to long-term patient discomfort or even complaints. The choice to perform additional buckle surgery in the cases presented here was based on the extent of retinal traction/retinal shortening and the behavior of the retinal tissue when PFO was filled into the vitreous cavity. Whether or not the encircling buckle would have been necessary in each of the cases to achieve stable reattachment (ie, using PFO alone) cannot be sufficiently answered. This is a shortcoming of this study and may only be identified in larger prospective series.

We have shown that a staged approach using PFO as a primary short-term endotamponade in a $23 \mathrm{G}$ PPV surgery approach allows for satisfactory management of complex $\mathrm{RD}$ with minimal complications and a comparable outcome compared with previous report in similar patients. The main advantages are the good predictability to reattach the retina, as well as the significantly reduced number of surgeries that are required to achieve retinal reattachment resulting in the long-term success rate as demonstrated by the long follow-up period in our study.

\section{Disclosure}

None of the authors have any proprietary/financial interests to disclose or is supported financially by any of the companies mentioned in the manuscript. 


\section{References}

1. Girard P, Mimoun G, Karpouzas I, Montefiore G. Clinical risk factors for proliferative vitreoretinopathy after retinal detachment surgery. Retina. 1994;14(5):417-424.

2. Charteris DG, Sethi CS, Lewis GP, Fisher SK. Proliferative vitreoretinopathy: developments in adjunctive treatment and retinal pathology. Eye (Lond). 2002;16(4):369-374.

3. Colyer MH, Chun DW, Bower KS, Dick JS, Weichel ED. Perforating globe injuries during operation Iraqi Freedom. Ophthalmology. 2008;115(11):2087-2093.

4. Laqua H, Machemer R. Glial cell proliferation in retinal detachment (massive periretinal proliferation). Am J Ophthalmol. 1975;80(4):602-618.

5. Machemer R, Laqua H. Pigment epithelium proliferation in retinal detachment (massive periretinal proliferation). Am J Ophthalmol. 1975;80(1):1-23.

6. Campochiaro PA, Jerdan JA, Glaser BM, Cardin A, Michels RG. Vitreous aspirates from patients with proliferative vitreoretinopathy stimulate retinal pigment epithelial cell migration. Arch Ophthalmol. 1985;103(9): 1403-1405.

7. Glaser BM, Cardin A, Biscoe B. Proliferative vitreoretinopathy. The mechanism of development of vitreoretinal traction. Ophthalmology. 1987;94(4):327-332.

8. Peyman GA, Smith RT, Charles H. Injection of fluorosilicone oil and pars plana vitrectomy for complex retinal detachment. Can J Ophthalmol. 1987;22(5):276-278.

9. Batman C, Cekic O. Effects of the long-term use of perfluoroperhydrophenanthrene on the retina. Ophthalmic Surg Lasers. 1998;29(2):144-146.

10. Viebahn M, Buettner H. Perfluorophenanthrene unsuitable for postoperative retinal tamponade. Am J Ophthalmol. 1994;118(1):124-126.

11. Gerding H, Kolck A. [Perfluorohexyloctane as internal tamponade in patients with complicated retinal detachment. Results after 6 months]. Ophthalmologe. 2004;101(3):255-262. German.

12. Roider J, Hoerauf H, Kobuch K, Gabel VP. Clinical findings on the use of long-term heavy tamponades (semifluorinated alkanes and their oligomers) in complicated retinal detachment surgery. Graefes Arch Clin Exp Ophthalmol. 2002;240(12):965-971.

13. Vote B, Wheen L, Cluroe A, Teoh H, McGeorge A. Further evidence for proinflammatory nature of perfluorohexyloctane in the eye. Clin Experiment Ophthalmol. 2003;31(5):408-414.
14. Mertens S, Bednarz J, Engelmann K. Evidence of toxic side effects of perfluorohexyloctane after vitreoretinal surgery as well as in previously established in vitro models with ocular cell types. Graefes Arch Clin Exp Ophthalmol. 2002;240(12):989-995.

15. Sandner D, Herbrig E, Engelmann K. High-density silicone oil (Densiron) as a primary intraocular tamponade: 12-month follow up. Graefes Arch Clin Exp Ophthalmol. 2007;245(8):1097-1105.

16. Theelen T, Tilanus MA, Klevering BJ. Intraocular inflammation following endotamponade with high-density silicone oil. Graefes Arch Clin Exp Ophthalmol. 2004;242(7):617-620.

17. Drury B, Bourke RD. Short-term intraocular tamponade with perfluorocarbon heavy liquid. Br J Ophthalmol. 2011;95(5):694-698.

18. Rush R, Sheth S, Surka S, Ho I, Gregory-Roberts J. Postoperative perfluoro-n-octane tamponade for primary retinal detachment repair. Retina. 2012;32(6):1114-1120.

19. Barthelmes D, Alexander S, Mitchell P, Chandra J. Hybrid 20/23-Gauge pars plana vitrectomy for retained lens fragments after cataract surgery. Retina. 2012;32(9):1749-1755.

20. Fannin LA, Schiffman JC, Budenz DL. Risk factors for hypotony maculopathy. Ophthalmology. 2003;110(6):1185-1191.

21. Ugahary LC, Ganteris E, Veckeneer M, et al. Topical ibopamine in the treatment of chronic ocular hypotony attributable to vitreoretinal surgery, uveitis, or penetrating trauma. Am J Ophthalmol. 2006;141(3):571-573.

22. Quiram PA, Gonzales CR, Hu W, et al. Outcomes of vitrectomy with inferior retinectomy in patients with recurrent rhegmatogenous retinal detachments and proliferative vitreoretinopathy. Ophthalmology. 2006; 113(11):2041-2047.

23. Rofail M, Lee LR. Perfluoro-n-octane as a postoperative vitreoretinal tamponade in the management of giant retinal tears. Retina. 2005;25(7):897-901.

24. Gerding H. Heavy internal tamponades and risk of hypotony. Eye (Lond). 2010;24(6):1114.

25. Romano MR, Groenwald C, Das R, Stappler T, Wong D, Heimann H. Removal of Densiron-68 with a 23-gauge transconjunctival vitrectomy system. Eye (Lond). 2009;23(3):715-717.
Clinical Ophthalmology

\section{Publish your work in this journal}

Clinical Ophthalmology is an international, peer-reviewed journal covering all subspecialties within ophthalmology. Key topics include: Optometry; Visual science; Pharmacology and drug therapy in eye diseases; Basic Sciences; Primary and Secondary eye care; Patient Safety and Quality of Care Improvements. This journal is indexed on Submit your manuscript here: http://www.dovepress.com/clinical-ophthalmology-journal

\section{Dovepress}

PubMed Central and CAS, and is the official journal of The Society of Clinical Ophthalmology (SCO). The manuscript management system is completely online and includes a very quick and fair peer-review system, which is all easy to use. Visit http://www.dovepress.com/ testimonials.php to read real quotes from published authors. 